\title{
Voltage Control Performance Evaluation using Synchrophasor Data
}

\author{
Christoph Lackner, Joe H. Chow \\ Rensselaer Polytechnic Institute \\ lacknc@rpi.edu, chowj@ rpi.edu
}

\author{
Felipe Wilches-Bernal \\ Sandia National Laboratories \\ fwilche@sandia.gov
}

\author{
Atena Darvishi \\ New York Power Authority \\ Atena.Darvishi@nypa.gov
}

\begin{abstract}
With increasing availability of synchrophasor technology, enabled by phasor measurement units (PMUs), applications based on this technology are being implemented as a practical approach for power systems monitoring and control. While synchrophasor data provides significant advantages over SCADA data it has limitations, especially in the area of model validation and estimation. With the increasing complexity of the power system, the need for equipment monitoring and performance evaluation becomes more relevant, traditionally model validation and estimation process can be used to look at control equipment performance. However, due to the challenges associated with these processes there are limitations on the performance evaluation. This work introduces am improved signal-processing based algorithm to monitor control system performance during disturbance events in the power system and during ambient conditions, or normal power system operation, additionally the algorithm is demonstrated on data obtained from the interconnection point of a STATCOM device and a synchronous generator during ambient and disturbance operation.
\end{abstract}

\section{Introduction}

Synchrophasor technology, enabled by phasor measurement units (PMUs), is now prevalent in power systems around the world. Applications based on this technology such as state estimation are being

This research was in part supported by the Engineering Research Center Program of the National Science Foundation and the Department of Energy under NSF Award Number EEC-1041877, NSF Award Number EEC-1550029, the CURENT Industry Partnership Program and NYSERDA under Award Number PON-3397. Sandia National Laboratories is a multimission laboratory managed and operated by National Technology and Engineering Solutions of Sandia, LLC., a wholly owned subsidiary of Honeywell International, Inc., for the U.S. Department of Energy's National Nuclear Security Administration under contract DE-NA0003525. This research was supported in part by the U.S. Department of Energy Transmission Reliability program. implemented as a practical approach for power systems monitoring and control $[1,2]$. One of the advantages of PMUs is, that they provide much higher data resolution than traditional SCADA measurements. This allows the data to be used for a variety of equipment monitoring and evaluation purposes, such as synchronous generator model validation and monitoring [3].

Voltage control and frequency control are two integral parts of keeping a power system stable. Voltage regulation is traditionally done through excitation systems on generators. However, with the increase of renewable resources and the advancements in power electronics, it becomes a possibility to provide voltage control, and primary frequency control through other means.

With the increase of the equipment that is capable of providing voltage and frequency control, it becomes critical to evaluate it and ensure the control functions are performed properly.

A significant amount of work has been done using PMU data for dynamic state estimation; in [4] this approach is used for synchronous generator model validation and identification. While PMUs nominally report data at 30 or 60 samples per second, most generator model calibration algorithms require much higher sampling rates [4]. This means PMUs with higher resolution need to be installed to monitor and evaluate generator control system performance.

Some previous work in $[5,6]$ has looked at using reduced-order models of power electronics equipment such as STATCOMs and VSCs which can be validated and estimated using lower resolution PMU data. However this work is limited to equipment connected to the power grid through power electronic interfaces and does not apply to traditional equipment such as synchronous generators.

This work introduces a reduced-order dynamic model of a control equipment similar to the one used in [7] to estimate and monitor control system performance without requiring higher sampling rates. The work in [7] focuses on the use of the reduced-order 
dynamical model in state estimation, while this paper introduces a reduced-order dynamic model of a voltage control equipment, such as a synchronous generators excitation system, that can be used to evaluate the equipment's performance based on PMU measurements at the current reporting rates. Previous work in [8] has focused on estimating a reduced-order dynamic model of a synchonous generator to estimate its voltage control performance. The work in [9] is focused on a reduced-order model to estimate the voltage control performance of a STATCOM.

In addition to introducing the reduced-order model, this work proposes a signal-processing based approach to estimate this model using data collected by a PMU at the point of interconnection (POI) of the equipment. The efficacy of the proposed algorithm is demonstrated using historical PMU measurements taken during disturbance events in the system as well as measurements taken during ambient operation.

The remainder of this paper is organized as follows. Section 2 introduces the reduced-order model. Section 3 proposes the signal-processing based algorithm used to estimate the reduced-order model from PMU data. Section 4 illustrates the proposed algorithm using historical PMU data from the New York Power System (NYPS). Finally, Section 5 summarizes the results and concludes the paper.

\section{Reduced-Order Dynamic Model}

Voltage control and frequency control are two integral parts of keeping a power system stable. This section introduces a reduced-order dynamic model, that can be used to model voltage controls. In [8] a similar model was introduced to estimate a synchronous generator's frequency and voltage control systems. The work in [9] also proposed a similar reduced-order model to evaluate the performance of a STATCOM. Section 2.1 introduces a mathematical description of the reduced-order model used in this work. Section 2.2 introduces phase plots, which can be used to visualize the droop and time constant of the reduced-order model.

\subsection{Model Description}

The model introduced in this section is of the same form for a STATCOM and a synchronous generator, and a detailed description of how the model can be obtained from a full STATCOM model or a full synchronous generator model can be found in [8] and [9], respectively. However, this work is not limited to any specific type of control equipment, but can be applied to any equipment designed to participate in voltage regulation. Fig. 1 shows a simplified voltage control block-diagram.

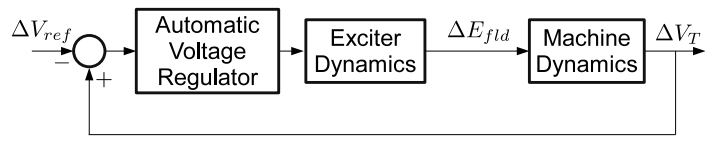

Figure 1: Simplified Voltage Regulation Feedback Loop.

Fig. 2a shows a synchronous generator connected to the power system. By monitoring the voltage at the point of interconnection (POI) and some flow variables such as the reactive power or current, it is possible to look at the synchronous generator model without considering the model of the connected power system. This can be used to develop a simplified dynamic model representing the generator, such as the one shown in Fig. 2b. The reactive and active power injections are modeled separately each consisting of a linear, time-invariant transfer function.

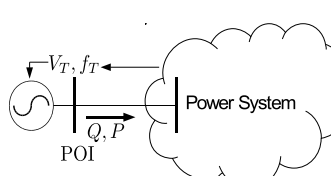

(a) Full POI Model

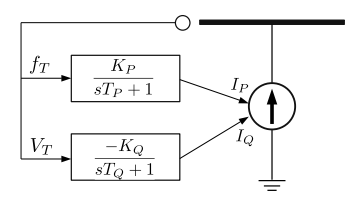

(b) Reduced-Order Model

Figure 2: Synchronous Machine Full Model and Reduced-Order Model.

When looking at a transient synchronous generator model the transient model is of order 9. In order to further reduce the complexity of the model a 1st-order transfer function is used to estimate the behavior of the model. The reactive injection of the generator is then given by:

$$
Q(s)=\frac{K_{Q V}}{T_{Q V} s+1} V(s)
$$

where $Q(s)$ and $V(s)$ are the Laplace transforms of the reactive power injections and the POI voltage, respectively.

The work in [9] and [8] shows a balanced model reduction based approach to reduce the full model of a STATCOM and a synchronous generator to the form (1).

For most control equipment the reduced-order model in (1) is only valid in a limited frequency range. Because the proposed algorithm uses the estimated 
reduced-model to characterize performance of the voltage and frequency control systems, this range should be selected accordingly.

Control system performance is based on parameters such as gains and time constants. For instance, in a synchronous machine exciter, modeling different control system performance is achieved by modifying the gain $K_{A}$ or the time constant $T_{A}$. Fig. 3 shows the effect these parameters have on the transfer function of a synchronous generator.

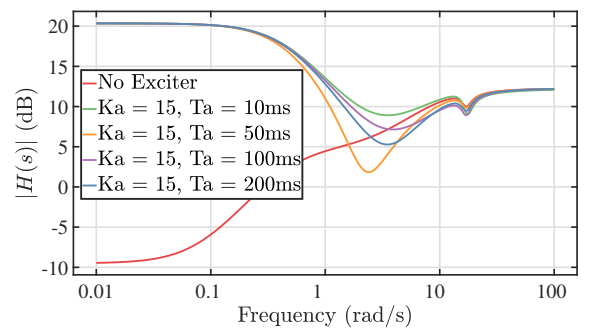

(a) Effect of $T_{A}$

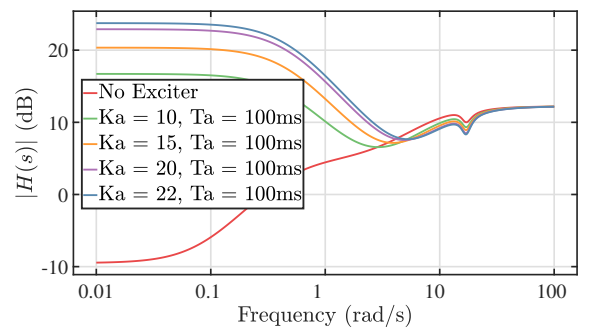

(b) Effect of $K_{A}$

Figure 3: Effect of the Exciter Parameters on Synchronous Generator Transfer Function.

Based on this figure the range of frequencies for which the model (1) should be valid is defined as $\omega<2$ $\mathrm{rad} / \mathrm{s}$. A similar analysis can be done for the control system of a STATCOM resulting in $\omega<5 \mathrm{rad} / \mathrm{s}$ [9].

\subsection{Phase Plots}

The effects of voltage controls are illustrated in Fig. 4 [10]. Figs. $4 a$ and $4 b$ show the voltage droop due to the regulation effect. The difference between these two responses is due to the time constant in the control loop. For example, a FACTS device can respond very quickly resulting in a straight-line response as shown in Fig. 4a, whereas a hydraulic or steam turbine would exhibit a loop-type behavior as shown in Fig. 4b.

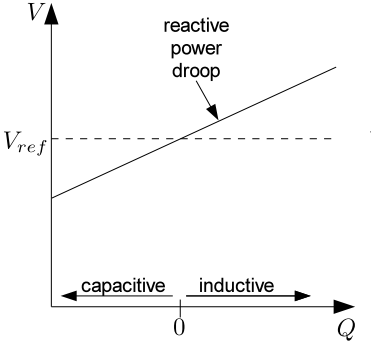

(a) Fast Regulation

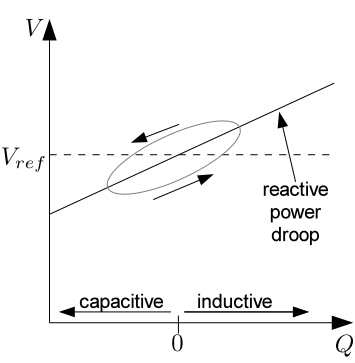

(b) Slow Regulation
Figure 4: Dynamic Voltage Control Phase Plot.

\section{Proposed Performance Evaluation Algorithm}

This section introduces the signal-processing based algorithm, which uses the POI reactive power injection measurement and the POI voltage measurement to find the estimated reduced-order dynamic model. Fig 5 shows the basic algorithm which consists of four stages:

1. Initial Data Processing

2. Dynamics Separation of the Signals

3. Frequency Component Selection

4. Dynamic Model Estimation

The algorithm estimates the gain and time constant of the estimated reduced-order dynamic model using POI synchrophasor measurements. Fig. 6 shows the measured voltage signal of a synchronous machine and the reactive power injection signal, as it is processed in each stage. The figure also shows some of the intermediate steps in each stage, which are explained in more detail in Sections 3.1 - 3.4. Figs. $6 \mathrm{a}$ and $6 \mathrm{~b}$ illustrate Stage 1 of the algorithm. Figs. $6 \mathrm{c}$ to $6 \mathrm{f}$ illustrate Stage 2 and Figs. $6 \mathrm{~g}$ and $6 \mathrm{~h}$ illustrate Stage 3.

Note that with the exception of the dynamic model estimation stage, each stage processes a signal independent of the other signals. To simplify the notation in the following sections, $x$ is used to refer to a single signal, which can be the voltage or reactive power signal.

\subsection{Initial Data Processing}

Fig. 7 shows an overview of this stage. First a missing data recovery algorithm is used to ensure there are no missing points.

The data recovery algorithm used in this work was introduced in [11]. Figs. $6 \mathrm{a}$ and $6 \mathrm{~b}$ show a voltage 


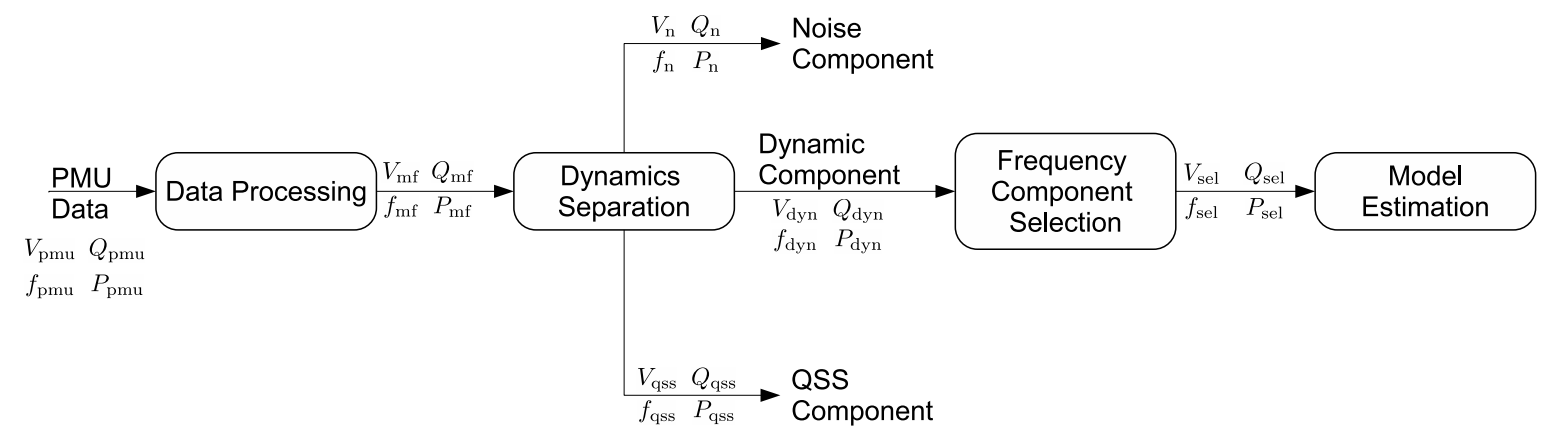

Figure 5: Performance Evaluation Algorithm Flow Chart.

and reactive power signal before and after the data recovery is applied. This is the only step in the entire algorithm that requires measurements beyond the POI of the equipment that is being evaluated.

After recovering any missing data, a second-order median filter is applied to remove any noise in the signal, as well as to ensure high frequency components are removed [12]. For simulated data, which contains no noise, this filter has no effect; however, for real PMU data such as the data in Figs. 6, the median filter acts as a smoothing operator.

The output of the second-order median filter is defined as

$$
x_{m f}[k]=\operatorname{median}\left(x_{\mathrm{rec}}[k-1], x_{\mathrm{rec}}[k], x_{\mathrm{rec}}[k+1]\right)
$$

Note that this filter is a non-linear filter. In [12] the authors show that this second-order filter can improve many synchrophasor-based applications while not significantly altering the information present in the signal.

\subsection{Dynamics Separation}

Power systems consist of a large number of interconnected generators and loads. Due to the stochastic nature of the loads and the need to balance load and generation in the system, the operating point of the system is continuously changing. Some of these changes are due to the control equipment in the system responding to changes in load and generation. However, some of these changes are related to other functions in the system such as economic dispatch, the intrinsic variability of some of the renewable generation such as wind or solar, or topology changes due to line switching. Since the algorithm is designed to monitor the performance of the control equipment, it is necessary to distinguish between the changes caused by the control equipment and those caused by other parts of system operation such as Automatic Generation Control [9]. This is achieved by separating the measurement signals into three distinct components,

$$
x_{\mathrm{mf}}(t)=x_{\mathrm{qss}}(t)+x_{\mathrm{dyn}}(t)+x_{\mathrm{n}}(t)
$$

where $x_{\mathrm{qss}}(t)$ is the quasi-steady-state (QSS) component or the slowly varying operating condition of the system, $x_{\mathrm{dyn}}(t)$ the dynamic component of the signal and $x_{\mathrm{n}}(t)$ the noise component.

The dynamic component contains the response of the control systems and is of interest for control systems evaluation. Practically this separation is done by the dynamics separation algorithm shown in Fig. 8. The advantage of this approach over some previous approaches to remove steady-state components, such as that described in [13], is that the dynamics separation algorithm in Fig. 8 is based on a non-linear approach. This allows the algorithm to compensate for some of the non-linearities present in the power system.

The first step of this algorithm is to apply a low-pass filter to the data. This filter removes any high-frequency noise that is present in the signal. The filter has a corner frequency of $10 \mathrm{~Hz}$ and is described by

$$
H(z)=\frac{a_{0}+a_{1} z^{-1}+a_{2} z^{-2}+a_{3} z^{-3}}{1+b_{1} z^{-1}+b_{2} z^{-2}+b_{3} z^{-3}}
$$

at a sampling rate of $30 \mathrm{~Hz}$ the coefficients are given by

$$
\begin{array}{ll}
a_{0}=0.3318 & \\
a_{1}=0.9954 & b_{1}=-0.9658 \\
a_{2}=0.9954 & b_{2}=-0.5826 \\
a_{3}=0.3318 & b_{3}=-0.1060
\end{array}
$$

The order of this filter was selected based on a trial and error approach. In order to evaluate different control 


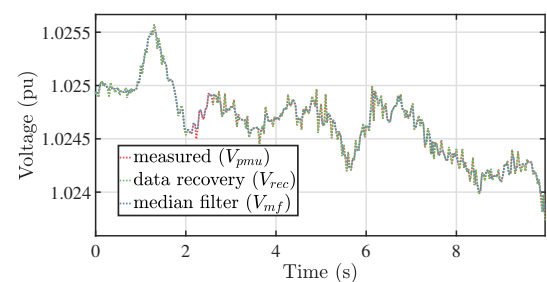

(a) Stage $1 V$ Signal

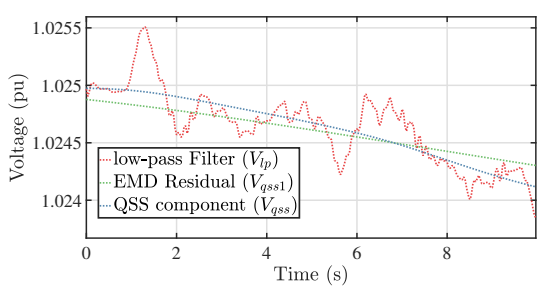

(c) Stage $2 V$ Signal

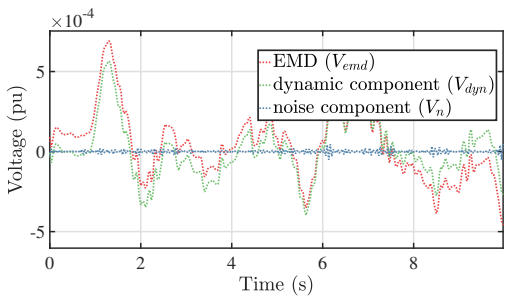

(e) Stage $2 V$ Signal

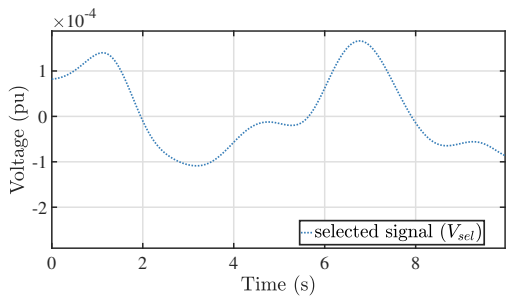

(g) Stage $3 V$ Signal

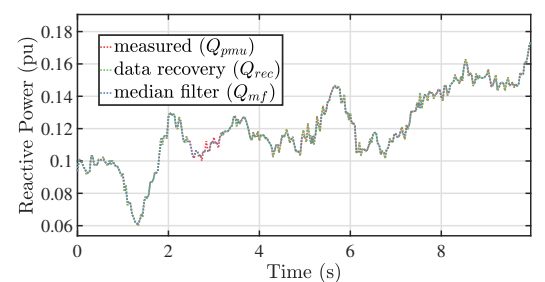

(b) Stage $1 Q$ Signal

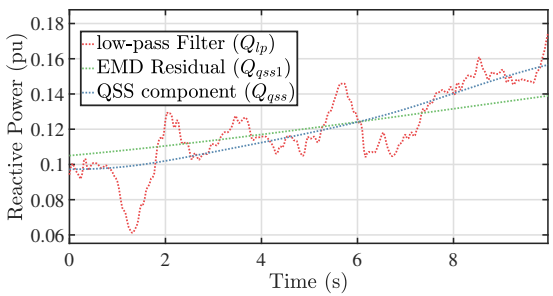

(d) Stage $2 Q$ Signal

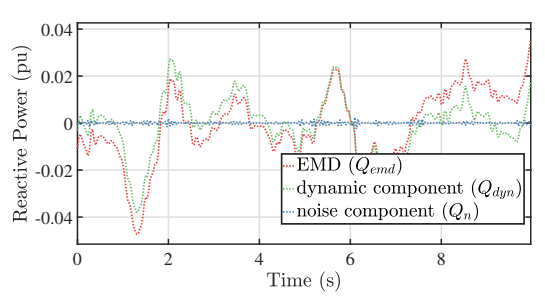

(f) Stage $2 Q$ Signal

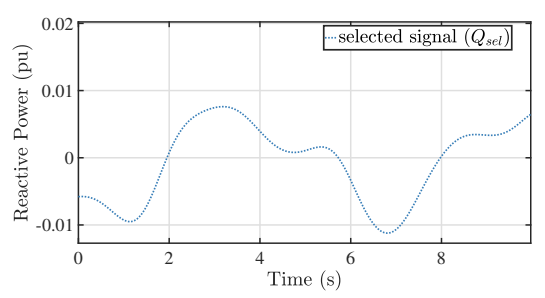

(h) Stage $3 Q$ Signal

Figure 6: Illustration of a Voltage Signal (Right Column) and Reactive Power Signal (Left Column) through Stages 1-3.

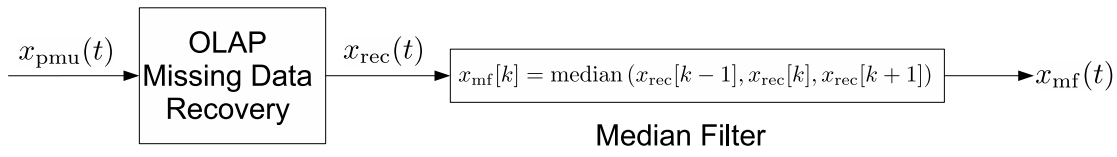

Figure 7: Initial Data Processing Stage Flowchart. 


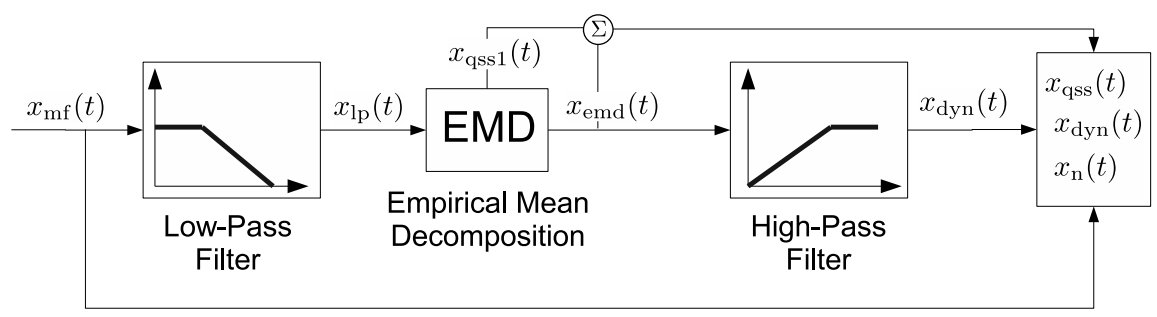

Figure 8: Dynamics Separation Stage Flowchart.

systems from those presented in this paper, the order of this filter can be adjusted.

Figs. $6 \mathrm{c}$ and $6 \mathrm{~d}$ show a voltage and a reactive power signal before and after the low-pass filter. Because of the high corner frequency, the filter does not have a significant effect on the signals. However, the filter does smooth out the signal slightly as seen in Fig. 9, which shows an expanded view of the signals in Fig. $6 \mathrm{c}$ and $6 \mathrm{~d}$.

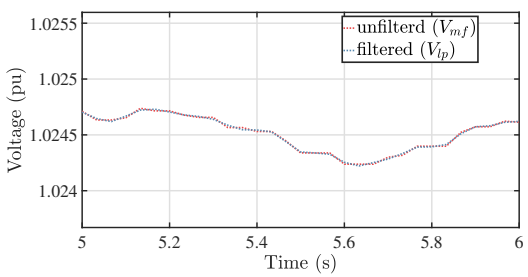

(a) Voltage Signal

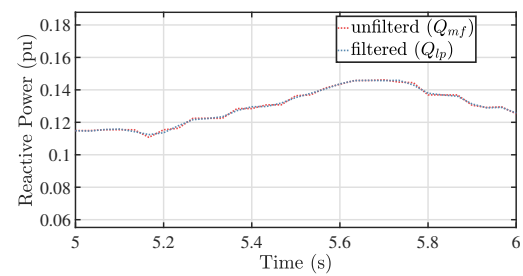

(b) Reactive Power Signal

Figure 9: Voltage and Reactive Power Signal before and after the Low-Pass Filter.

Next an Empirical Mean Decomposition (EMD) is performed to decompose the signal into two parts. EMD decomposes an arbitrary signal $x_{\mathrm{lp}}(t)$ into a number of Intrinsic Mode Functions (IMF) and a residual

$$
x_{\mathrm{lp}}(t)=\sum_{i=1}^{N} c_{i}(t)+r(t)
$$

where $c_{i}(t)$ are IMFs with variable frequency and amplitude, and $r(t)$ is the residual. By design, IMFs are nearly orthogonal and the sum of all of them contains almost the entire modal information present in the original signal [14].

Since changes in operating conditions due to highly non-linear processes such as economic dispatch, or stochastic processes like load changes, are not related to the modes of the system, part of the QSS component is given by

$$
x_{\mathrm{qss} 1}(t)=r(t)
$$

Thus the dynamic component of the original signal can be expressed as

$$
x_{\mathrm{emd}}(t)=\sum_{i=1}^{N} c_{i}(t)
$$

Fig. 6 illustrates this process using a voltage and a reactive power signal. Figs. $6 \mathrm{c}$ and $6 \mathrm{~d}$ show the signal before the EMD is applied ( $V_{l p}$ and $Q_{l p}$, respectively), as well as the residual $\left(V_{q s s 1}\right.$ and $\left.Q_{q s s 1}\right)$. Figs. 6e and 6f show the dynamic portion $\left(V_{e m d}\right.$ and $\left.Q_{e m d}\right)$.

Next a high-pass filter is used to remove any leftover DC bias from the dynamic component. This filter has a corner frequency of $0.001 \mathrm{~Hz}$ and is given by:

$$
H(z)=\frac{a_{0}+a_{1} z^{-1}+a_{2} z^{-2}+a_{3} z^{-3}}{1+b_{1} z^{-1}+b_{2} z^{-2}+b_{3} z^{-3}}
$$

and at a sampling rate of $30 \mathrm{~Hz}$ the coefficients are

$$
\begin{array}{ll}
a_{0}=1.000 & \\
a_{1}=-2.999 & b_{1}=3.000 \\
a_{2}=2.999 & b_{2}=-2.999 \\
a_{3}=-1.000 & b_{3}=1.000
\end{array}
$$

Similar to the low-pass filter in 4 , this is a 3rd-order filter and the order was selected based on a trial and error approach.

Figs. $6 \mathrm{e}$ and $6 \mathrm{f}$ show the signal before the high-pass filter is applied ( $V_{\text {emd }}$ and $\left.Q_{\text {emd }}\right)$, as well as the signal after the filter $\left(V_{\mathrm{dyn}}\right.$ and $\left.Q_{\mathrm{dyn}}\right)$. Because of the low 
corner frequency the filter has very little effect on the signals besides removing the DC offset. Finally the three components of the signal are computed as

$$
\begin{aligned}
x_{\mathrm{qss}} & =x_{\mathrm{qss} 1}+x_{\mathrm{emd}}-x_{\mathrm{dyn}} \\
x_{\mathrm{dyn}} & =x_{\mathrm{dyn}} \\
x_{\mathrm{n}} & =x_{\mathrm{mf}}-x_{\mathrm{qss}}-x_{\mathrm{dyn}}
\end{aligned}
$$

where $x_{\mathrm{emd}}$ and $x_{\mathrm{qss} 1}$ are the sum of IMFs and the residual of the EMD respectively. The next stage of the performance evaluation algorithm only uses the dynamic component $X_{\mathrm{dyn}}$.

\subsection{Frequency Component Selection}

The third stage of the algorithm is designed to ensure only the relevant frequency range is used to estimate the reduced-order dynamic model. Since the range is generally of the form $\omega<\omega_{c}$, as discussed in Section 2, a low-pass filter is sufficient to remove any components outside this range.

After selecting the desired frequency range, and the corresponding $\omega_{c}$, a third-order Butterworth filter with a cut-off frequency of $\omega_{c}$ is designed.

Fig. 6 shows the effect of this filter on a voltage and a reactive power signal. Figs. $6 \mathrm{e}$ and $6 \mathrm{f}$ show the signals before the filter is applied ( $V_{\mathrm{dyn}}$ and $\left.Q_{\mathrm{dyn}}\right)$ and Figs. $6 \mathrm{~g}$ and $6 \mathrm{~h}$ show the signals after the filter is applied $\left(V_{\text {sel }}\right.$ and $\left.Q_{\text {sel }}\right)$. Note that in this illustration the equipment is a synchronous generator excitation system, which means $\omega_{c}=2 \mathrm{rad} / \mathrm{s}$.

\subsection{Model Estimation}

In Stage 4 of the algorithm the processed and filtered signals are used to estimate the voltage response gain and time constant. Numerical optimization is used to minimize the mean-squares error (MSE) between the measured power output and the modeled power output. The modeled output is obtained by replaying the voltage as an input to the reduced-order model. The MSE is given by

$$
E=\int\left(Q_{\mathrm{sel}}(t)-\hat{Q}_{\mathrm{sel}}(t)\right)^{2} d t
$$

where $\hat{Q}_{\text {sel }}(s)$ and $V_{\text {sel }}(s)$, the Laplace transforms of $\hat{Q}_{\text {sel }}$ and $V_{\text {sel }}$, respectively, are related by

$$
\hat{Q}_{\mathrm{sel}}(s)=\frac{K}{T s+1} V_{\mathrm{sel}}(s)
$$

Since the MSE is not guaranteed to be globally convex, the optimization can run into numerical issues and converge to the local minimum instead of a global minimum. To avoid this issue, a reasonable starting point for the time constants and gains has to be selected. In general, the previous results can be used as a reasonable starting point. If the MSE converges to a local minimum, it will most likely be very different from the MSE obtained for the same equipment using a different dataset. This case is also an indication that the parameters of the control equipment have changed and can be used to flag the equipment for additional investigation.

The time constants and gains obtained from this estimation form the reduced-order dynamic model. By comparing these models over time, a change in the control system performance can be detected and the equipment can be flagged for additional investigation by the equipment owner or system operator.

Note that the MSE depends largely on the gain $K$ and is not significantly affected by small changes in $T$. Therefore the numeric minimization algorithm will focus on adjusting $K$ before adjusting $T$. Due to reaching a set maximum number of interpretations the algorithm might stop before finding the optimal $T$, resulting in a wider range of time constants than gains. While a comparison of $T$ can still yield some insight into the control system performance, it should be noted that the relative range of $T$ can vary significantly more than the relative range of $K$.

\section{Performance Evaluation Results}

This section introduces some results obtained when applying the proposed algorithm to historical PMU data. The algorithm described in Section 3 is applied to 30 seconds of PMU data taken at the POI of a STATCOM and a hydraulic generator in the New York Power system. Section 4.1 discusses the results obtained during disturbance events in the power system, while Section 4.2 describes the results obtained during ambient operation.

\subsection{Disturbance Data Results}

This section describes the results obtained during 16 generator trips in the eastern interconnection. All trips where larger than $900 \mathrm{MW}$ and located outside the NYPS.

The first control system analyzed was the voltage control of a STATCOM. Table 1 shows the estimated droop $\left(D=\frac{1}{K}\right)$ and time constant during each event.

As mentioned in Section 3.4, the time constant varies significantly more than the estimated droop due to numerical difficulties of estimating the correct time constant. 
Table 1: STATCOM Disturbance Data Performance Results.

\begin{tabular}{|c|c|c|}
\hline Event & Droop (\%) & T (ms) \\
\hline 1 & 3.175 & 106.06 \\
2 & 2.884 & 4.08 \\
3 & 3.217 & 3.88 \\
4 & 2.883 & 88.11 \\
5 & 2.886 & 4.61 \\
6 & 3.190 & 3.45 \\
7 & 2.845 & 3.64 \\
8 & 5.396 & 240.43 \\
9 & 2.738 & 5.05 \\
10 & 6.901 & 68.34 \\
11 & 3.134 & 26.77 \\
12 & 2.997 & 117.04 \\
13 & 2.736 & 5.00 \\
14 & 2.873 & 3.95 \\
15 & 3.045 & 59.26 \\
16 & 3.228 & 3.81 \\
\hline
\end{tabular}

Table 1 shows that with exception of Events 8 and 10, all results have a similar droop estimate. The equipment owner confirmed correct operation of the STATCOM during all events with the exception of Events 8 and 10. There are two identical STATCOM banks at the substation. If one bank of the STATCOM is out of service, the droop will effectively deteriorate to about $6 \%$, cutting the voltage regulation capability in half.

Fig. 10a shows a STATCOM phase plots similar to that in Fig. 4a. Figs. 10c and 10d show the measured voltage and reactive power output for comparison.

Fig. 10b shows the importance of Stages 1-3 of the proposed algorithm. Fig. 10b shows the same voltage control phase plots as Fig. 10a using the measured signals without applying the proposed algorithm. For the STATCOM the droop could be identified in the unfiltered data. However, the time constant cannot be identified properly since the measured data results in a much wider oval than the processed data in Fig. 10b.

The second control system analyzed was the voltage control of a hydraulic generator. Table 2 shows the estimated droops and time constants during each event. Due to data quality issues only a subset of events could be analyzed for these control systems.

Note that for the generator voltage control the droop is generally higher than for the STATCOM, implying less responsive voltage control. With the exception of Event 1, the droop is consistently between $10 \%$ and $20 \%$. While the time constant varies significantly it is in general higher than the time constants estimated for the STATCOM. Since the voltage control of a synchronous
Table 2: Synchronous Generator Control Performance during Disturbances.

\begin{tabular}{|c|c|c|}
\hline Event & Droop (\%) & $\mathrm{T}(\mathrm{ms})$ \\
\hline 1 & 30.533 & 1904.50 \\
2 & 17.929 & 4761.68 \\
3 & 18.917 & 4305.19 \\
4 & 17.281 & 5086.73 \\
5 & 16.770 & 506.72 \\
6 & 10.170 & 491.00 \\
7 & 10.799 & 1646.64 \\
8 & 18.749 & 874.44 \\
\hline
\end{tabular}

machine has to be achieved via the flux linkages in the machine, the effective voltage control is slower than that of a STATCOM which is connected to the power system through a power electronics interface. Thus the effective time constant of voltage control through a synchronous machine is longer.

Fig. 11 shows an example of the phase plots for the generator's voltage control. Similar to the STATCOM it is very difficult to estimate a droop-line based on the unfiltered data.

\subsection{Ambient Data Results}

This section describes the results obtained from 15 data sets taken during ambient conditions. The same STATCOM and generator as in Section 4.1 are analyzed.

Table 3 shows the STATCOM's estimated droop and time constant during the ambient data sets. Data sets 8 and 10 were taken shortly after Events 8 and 10 in Section 4.1, meaning the STATCOM was not operating as expected.

Table 3: STATCOM Ambient Data Performance Results.

\begin{tabular}{|c|c|c|}
\hline Dataset & Droop (\%) & T (ms) \\
\hline 1 & 2.652 & 20.28 \\
2 & 2.847 & 42.89 \\
3 & 3.469 & 27.91 \\
4 & 3.809 & 3.55 \\
5 & 3.212 & 4.04 \\
6 & 3.589 & 1.53 \\
7 & 2.844 & 3.54 \\
8 & 7.128 & 32.64 \\
9 & 2.836 & 24.00 \\
10 & 4.697 & 0.76 \\
11 & 2.005 & 4.70 \\
12 & 3.123 & 47.30 \\
13 & 2.620 & 4.01 \\
14 & 3.489 & 2.58 \\
15 & 2.768 & 3.80 \\
\hline
\end{tabular}




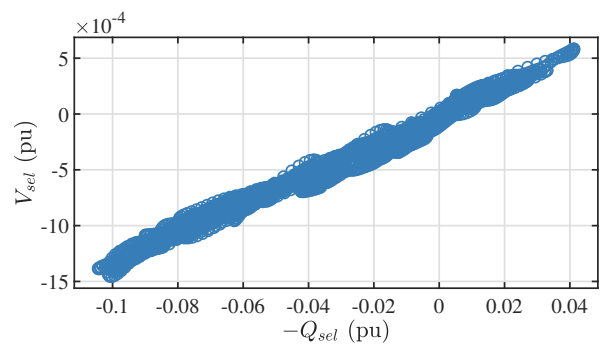

(a) Phase Plot

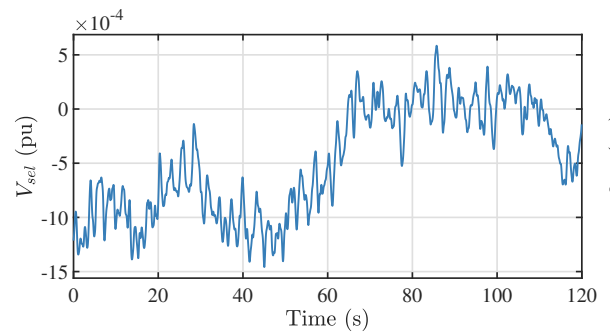

(c) $V$

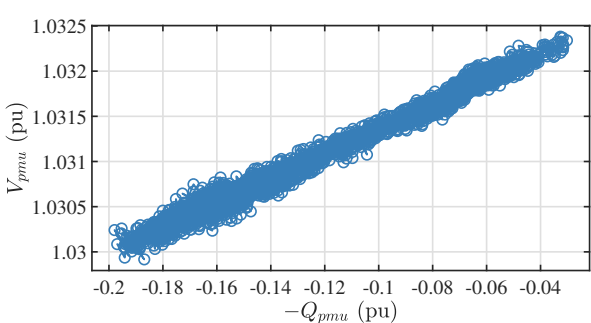

(b) Phase Plot unfiltered

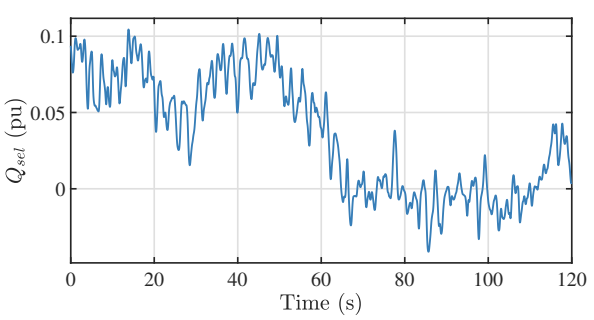

(d) $Q$

Figure 10: STATCOM POI Measurements during Disturbance Event.

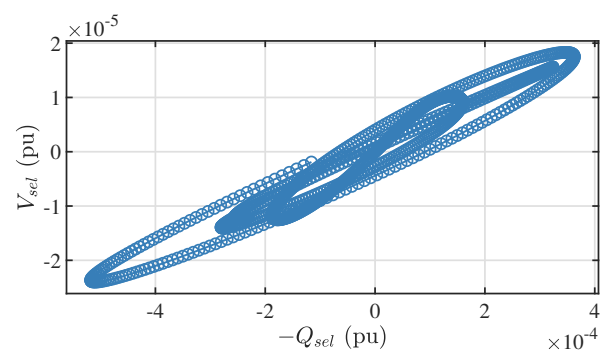

(a) $Q-V$

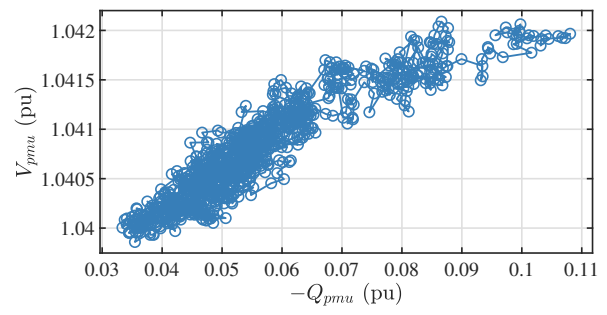

(b) $Q-V$ unfiltered

Figure 11: Generator Voltage Control Phase Plots during Disturbance Event.

Table 4 shows the droop and time constant estimates for the generators voltage control.

Fig. 12 shows a STATCOM phase plot using the filtered and unfiltered Data. During ambient power system operations it is very difficult to estimate droop based on the unfiltered data since the change in voltage is significantly smaller than during the disturbance events.
Table 4: Generator Ambient Data Performance Results.

\begin{tabular}{|c|c|c|}
\hline Dataset & Droop (\%) & $\mathrm{T}(\mathrm{ms})$ \\
\hline 1 & 6.514 & 561.73 \\
2 & 12.161 & 509.35 \\
3 & 20.055 & 1477.96 \\
4 & 15.076 & 1033.97 \\
5 & 18.029 & 989.40 \\
\hline
\end{tabular}

Fig. 13a the voltage control phase plot of a generator. Fig. 13b shows the same phase plot using unfiltered data.

\section{Conclusion}

This paper introduces a signal processing-based algorithm to estimate a simplified dynamic model of a voltage control equipment based on the point of interconnection measurements. The estimated dynamic model can be used to evaluate the performance of the equipment compared to historical performance and identify any changes in control system parameters. The work includes some historic performance evaluation results based on disturbance data as well as ambient data. The results obtained using ambient data match the results obtained during disturbance events reasonable well, which indicates the proposed algorithm could be used in real time continuously, without requiring a large power system disturbance. Since ambient data is constantly available this greatly improves the usefulness of this algorithm. 


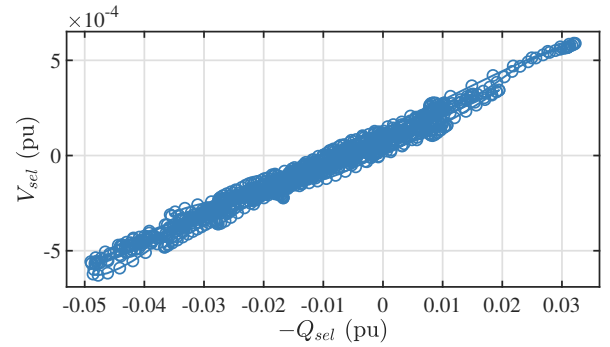

(a) $Q-V$

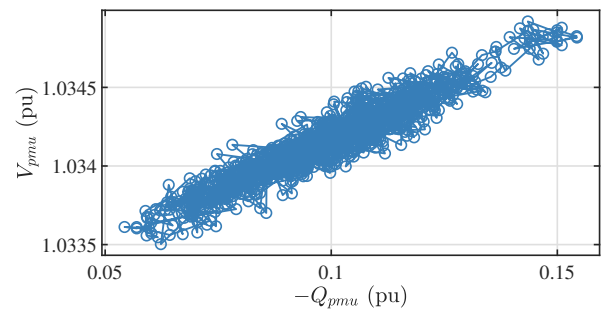

(b) $Q-V$ unfiltered

Figure 12: STATCOM Control Phase Plots during Ambient Conditions.

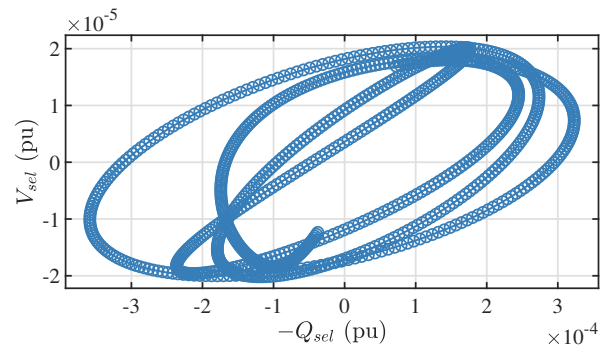

(a) $Q-V$

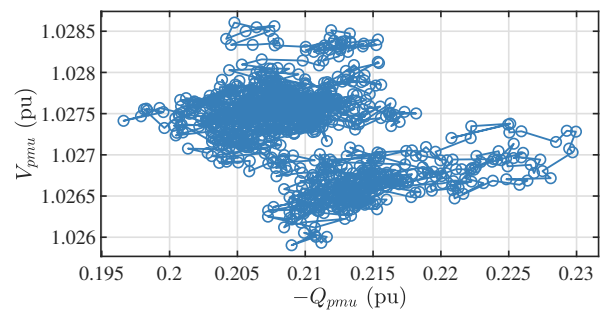

(b) $Q-V$ unfiltered

Figure 13: Generator Voltage Control Phase Plots during Ambient Conditions.

Future work includes the use of the proposed algorithm to monitor frequency control, as well as the adaption of the algorithm to continuously monitor control performance. In addition some of the insights gained in the relation between POI measurements and controller performance can be used in forced oscillation detection.

\section{References}

[1] S. G. Ghiocel et al., "Phasor-Measurement-Based State Estimation for Synchrophasor Data Quality Improvement and Power Transfer Interface Monitoring," IEEE Transactions on Power Systems, vol. 29, pp. 881-888, March 2014.

[2] C. Lackner, Q. Zhang, and J. H. Chow, "Real-Time phasor-only state estimation with topology processing as OpenPDC adapter," in 2017 IEEE Power Energy Society General Meeting, pp. 1-5, July 2017.

[3] Y. Li et al., "An innovative software tool suite for power plant model validation and parameter calibration using PMU measurements," in 2017 IEEE Power Energy Society General Meeting, pp. 1-5, July 2017.

[4] L. Sun, A. P. S. Meliopoulos, Y. Liu, and B. Xie, "Dynamic state estimation based synchronous generator model calibration using PMU data," in 2017 IEEE Power Energy Society General Meeting, pp. 1-5, July 2017.

[5] X. Jiang et al., "A Novel Approach for Modeling Voltage-Sourced Converter-Based FACTS Controllers," IEEE Transactions on Power Delivery, vol. 23, pp. 2591-2598, Oct 2008.

[6] C. Lackner, J. H. Chow, and F. Wilches-Bernal, "Performance Evaluation of STATCOM Equipment using Ambient and Disturbance Data," in Powertech, pp. 1-5, July 2019.

[7] W. Li, L. Vanfretti, and J. H. Chow, "Pseudo-dynamic network modeling for pmu-based state estimation of hybrid ac/dc grids," IEEE Access, vol. 6, pp. 4006-4016, 2018.

[8] C. Lackner, J. H. Chow, and F. Wilches-Bernal, "Estimation of Generator Control System Performance using Synchrophasor Data," in XIV SEPOPE, pp. 1-12, September 2018.

[9] C. Lackner and J. H. Chow, "Wide-Area Automatic Generation Control between Control Regions with High Renewable Penetration," in 2017 IREP, pp. 1-5, July 2017.

[10] P. W. Sauer, M. A. Pai, and J. H. Chow, Power System Dynamics and Stability with Synchrophasor Measurement and Power System Toolbox. Wiley-IEEE press, 3rd ed., 2017.

[11] G. M. De Mijolla, S. Konstantinopoulos, P. Gao, J. H. Chow, and M. Wang, "An evaluation of algorithms for synchrophasor missing data recovery," in 2018 Power Systems Computation Conference (PSCC), pp. 1-6, June 2018.

[12] I. Ivanov and A. Murzin, "Optimal filtering of synchronized current phasor measurements in a steady state," in 2015 IEEE International Conference on Industrial Technology (ICIT), pp. 1362-1367, March 2015.

[13] N. Zhou, J. W. Pierre, and J. F. Hauer, "Initial results in power system identification from injected probing signals using a subspace method," IEEE Transactions on Power Systems, vol. 21, pp. 1296-1302, Aug 2006.

[14] N. E. Huang et al., "The empirical mode decomposition and the hilbert spectrum for nonlinear and non-stationary time series analysis," Proceedings of the Royal Society of London. Series A: Mathematical, Physical and Engineering Sciences, vol. 454, no. 1971, pp. 903-995, 1998. 